\title{
Signatures of molecular single-particle states by level crossings in heavy ion collisions
}

\author{
Jae Y. Park \\ Department of Physics, North Carolina State University, Raleigh, North Carolina, 27650 \\ Walter Greiner \\ Institut für Theoretische Physik der Johann Wolfgang Goethe-Universität, Frankfurt am Main, Germany \\ Werner Scheid \\ Institut für Theoretische Physik der Justus-Liebig-Universität, Giessen, Germany \\ (Received 12 April 1979)
}

\begin{abstract}
In heavy ion collisions, the molecular single-particle motion may cause specific structures in the energy dependence of the cross sections which arise by the promotion of nucleons at level crossings according to the Landau-Zener excitation mechanism. In order to examine this effect in asymmetric heavy ion collisions, we have calculated level diagrams of the two-center shell model for the target projectile combinations ${ }^{13} \mathrm{C}+{ }^{16} \mathrm{O}$ and ${ }^{12} \mathrm{C}+{ }^{17} \mathrm{O}$ and analyzed with respect to inelastic excitation and neutron transfer. We select certain reactions as possible candidates for showing enhanced cross sections for nucleon excitation and transfer due to real and avoided level crossings near the Fermi level.
\end{abstract}

[NUCLEAR REACTIONS Heavy ion scattering, molecular theory of nucleon] transfer, asymmetric two-center shell model; ${ }^{13} \mathrm{C}\left({ }^{16} \mathrm{O},{ }^{17} \mathrm{O}\right)$, calculated level diagrams.

\section{INTRODUCTION}

For a microscopic description of fission and heavy ion collisions two-center shell models (TCSM) have been developed and applied. ${ }^{1-3}$ The TCSM are based on the assumption that the nucleons can be described with molecular states during the heavy ion reaction. This means that the motion of the nuclear centers has to be adiabatically slow compared with the rearrangement of the mean field of nucleons. Nucleus-nucleus collisions do not completely satisfy the condition of adiabaticity since the characteristic reaction and rearrangement times are of the same order of magnitude. Therefore, the molecular model for nucleons remains mainly a theoretical concept as long as no unique experimental signatures for molecular single-particle states become available. A survey of the experimental situation is summarized in Ref. 4.

In this paper we study the possibility of detecting special signatures of molecular single-particle states in inelastic and transfer heavy ion cross sections. One expects enhancements and structures in the energy dependence of cross sections due to the Landau-Zener effect at points of real and avoided crossings of the molecular levels in the TCSM. ${ }^{5}$ Such enhancements and structures are well known in atomic collisions and are explained by Fano and Lichten ${ }^{6}$ by the effect that electrons in molecular orbital levels are promoted to higher states mainly near points of real and avoided level crossings. For nucleus-nucleus collisions Glas and Mosel $^{7}$ have calculated the excitation of the compound nucleus in the ${ }^{16} \mathrm{O}-{ }^{16} \mathrm{O}$ scattering by applying the Landau-Zener formula to the level diagram of the symmetric TCSM. They found that the main excitation process in heavy ion collisions is due to the promotion of nucleons at points of level crossings.

A first step to obtain cross sections for molecular single-particle transitions is the calculation of realistic two-center level diagrams. Since symmetric systems, such as ${ }^{16} \mathrm{O}+{ }^{16} \mathrm{O}$, show only a very few level crossings near the Fermi level, we investigate level diagrams of asymmetric systems such as ${ }^{13} \mathrm{C}+{ }^{16} \mathrm{O}$ and ${ }^{12} \mathrm{C}+{ }^{17} \mathrm{O}$. In Sec. II we present calculations of diagrams for the neutron levels, carried out with the asymmetric TCSM, and adjust the various parameters with the experimental single-particle energies of the nuclei under consideration. In Sec. III we relate the level diagrams to inelastic excitations and neutron transfer reactions. Several reaction channels are found where level crossings occur which may cause structures in the energy dependence of the cross section. The measurements of these selected reactions could possibly give an answer about the existence of molecular single-particle levels in heavy ion collisions.

\section{LEVEL DIAGRAMS IN THE ASYMMETRIC TWO-CENTER SHELL MODEL}

We examine the possibility of the nuclear Landau-Zener mechanism in asymmetric heavy ion collisions. To do this we calculate single-particle 

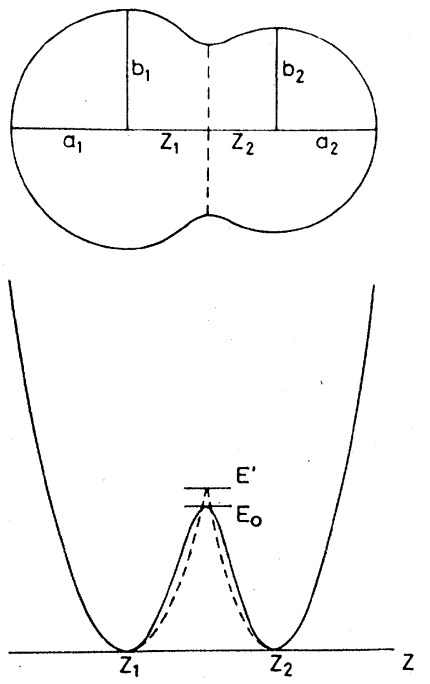

FIG. 1. The potential of the asymmetric TCSM along the $z$ axis and the associated nuclear shape.

energies as a function of the separation distance between two colliding nuclei. As a shell model we use the asymmetric two-center shell model (ATCSM) which has been applied by Maruhn and Greiner $^{3}$ to study nuclear fission and HI scattering. The potential along the $z$ axis and the associated nuclear shape are shown in Fig. 1.

The single-particle Hamiltonian of the ATCSM is given by $^{3}$

$$
\begin{aligned}
H= & -\frac{\hbar^{2}}{2 m} \Delta+V(\rho, z)+V_{\overrightarrow{1} \vec{s}}(\overrightarrow{\mathrm{r}}, \overrightarrow{\mathrm{p}}, \overrightarrow{\mathrm{s}}) \\
& +V_{l} 2(\overrightarrow{\mathrm{r}}, \overrightarrow{\mathrm{l}})-V_{0},
\end{aligned}
$$

where

$$
V(\rho ; z)=\left\{\begin{array}{c}
\frac{1}{2} m \omega_{z i}{ }^{2} z^{\prime 2}+\frac{1}{2} m \omega_{\rho i}{ }^{2} \rho^{2}, \\
z<z_{1} \text { for } i=1 \text { and } z>z_{2} \text { for } i=2 \\
\frac{1}{2} f m \omega_{z i}{ }^{2} z^{\prime 2}\left(1+c_{i} z^{\prime}+d_{i} z^{\prime 2}\right) \\
+\frac{1}{2} m \omega_{\rho i}{ }^{2}\left(1+g_{i} z^{\prime 2}\right) \rho^{2}, \\
z_{1}<z<0 \text { for } i=1 \text { and } 0<z<z_{2} \text { for } i=2
\end{array}\right.
$$

with

$$
z^{\prime}= \begin{cases}z-z_{1}, & z<0 \\ z-z_{2}, & z>0 .\end{cases}
$$

The barrier parameter $\epsilon$ is defined by the potential barriers, as shown in Fig. 1:

$$
\epsilon=E_{0} / E^{\prime} \text {. }
$$

The deformation parameters $\beta_{i}$ are defined by the lengths of the axes (see also Fig. 1):

$$
\beta_{i}=a_{i} / b_{i}, \quad i=1,2 .
$$

The single-particle energies depend on the internuclear distance $R=z_{2}-z_{1}$ and on the frequencies $\omega_{z i}, \omega_{\rho i}$ of the potential. In adiabatic collisions the frequencies have to be adjusted for each internuclear distance in order that the volume enclosed in an equipotential surface remains constant (volume conservation). The parameters of the two-center potential are determined by fitting the experimental single-particle levels near the Fermi surface of the separated nuclei $(R \rightarrow \infty)$ and the compound nucleus $(R \rightarrow 0)$. The energy eigenvalues of the two-center shell model with $\beta_{i}=0$ are given for $R=0$ and $R \rightarrow \infty$ by

$$
\begin{aligned}
E= & \left(N+\frac{3}{2}\right) \hbar \omega-V_{0}+a\langle\overrightarrow{\mathbf{l}} \cdot \overrightarrow{\mathbf{s}}\rangle \\
& +b\left(\left\langle l^{2}\right\rangle-\frac{1}{2} N(N+3)\right),
\end{aligned}
$$

where $N=2(n-1)+l, \hbar \omega=C A^{-1 / 3}$. It yields

$$
\begin{aligned}
E(n l j)= & \left(N+\frac{3}{2}\right) \hbar \omega-V_{0}+0.5 a\left(j(j+1)-l(l+1)-\frac{3}{4}\right) \\
& +b\left(l(l+1)-\frac{1}{2} N(N+3)\right) .
\end{aligned}
$$

Since we want to discuss the reactions ${ }^{13} \mathrm{C}+{ }^{16} \mathrm{O}$ and ${ }^{12} \mathrm{C}+{ }^{17} \mathrm{O}$ in the following, we list the experimental neutron single-particle energies of ${ }^{13} \mathrm{C}$, ${ }^{17} \mathrm{O}$, and ${ }^{29} \mathrm{Si}$ in Table I. These energies are used to fit the parameters $a, b, C$, and $V_{0}$ of Eq. (6). The parameters, listed in Table II, are inserted into the ATCSM and interpolated for finite values of $R$. The parameter sets I and II of Table II have the same value $V_{0}$ for the compound nucleus and the separated nuclei in contrast to set III, where in addition the $1 f_{7 / 2}$ level of ${ }^{29} \mathrm{Si}$ has been fitted. The deformation parameters $\beta_{i}$ are set $\beta_{i}=1$ (no deformation) and the barrier parameter $\epsilon=0.68$ in order to obtain a proper asymptotic convergence of the magnetic sublevels.

Figures 2-4 show the neutron level structure of the ATCSM for the reactions ${ }^{13} \mathrm{C}+{ }^{16} \mathrm{O} \rightarrow{ }^{29} \mathrm{Si}$ and ${ }^{12} \mathrm{C}+{ }^{17} \mathrm{O} \rightarrow{ }^{29} \mathrm{Si}$. For the internuclear distance $R=0$ we recognize the single-particle states of the compound nucleus ${ }^{29} \mathrm{Si}$ and for $R \rightarrow \infty$ the singleparticle states of the separated nuclei ${ }^{12,13} \mathrm{C}$ and ${ }^{16,17} \mathrm{O}$. The level diagrams differ mainly in the

TABLE I. Experimental neutron single-particle energies (Ref. 20) used in the fits of the parameters of the asymmetric TCSM (in MeV).

\begin{tabular}{crrrrr}
\hline \hline Nucleus & $1 p_{1 / 2}$ & $1 d_{5 / 2}$ & $2 s_{1 / 2}$ & $1 d_{3 / 2}$ & $1 f_{\eta / 2}$ \\
\hline${ }^{13} \mathrm{C}$ & -4.95 & -1.09 & -1.86 & & \\
${ }^{17} \mathrm{O}$ & & -4.14 & -3.27 & 0.943 & \\
${ }^{29} \mathrm{Si}$ & & -10.50 & -8.47 & -7.20 & -4.85 \\
\hline \hline
\end{tabular}


TABLE II. Parameters of the ATCSM potential for $R=0$ and $R=\infty$ (in MeV). The parameters $\hbar \omega, a, b$, and $V_{0}$ are defined in Eqs. (5) and (6).

\begin{tabular}{|c|c|c|c|c|c|c|c|c|}
\hline No. & Reaction & $C=\hbar \omega A^{1 / 3}$ & Nucleus & $\hbar \omega$ & $a$ & $b$ & $V_{0}$ & Fitted levels \\
\hline I & ${ }^{13} \mathrm{C}+{ }^{16} \mathrm{O} \rightarrow{ }^{29} \mathrm{Si}$ & 39.47 & $\begin{array}{l}{ }^{13} \mathrm{C} \\
{ }^{16} \mathrm{O} \\
{ }^{29} \mathrm{Si}\end{array}$ & $\begin{array}{l}16.79 \\
15.66 \\
12.85\end{array}$ & $\begin{array}{l}-7.123 \\
-5.723 \\
-1.320\end{array}$ & $\begin{array}{r}1.315 \\
0.809 \\
-0.118\end{array}$ & $\begin{array}{l}54.05 \\
54.05 \\
54.05\end{array}$ & $\begin{array}{l}1 p_{1 / 2}, 1 d_{5 / 2}, 2 s_{1 / 2} \\
1 d_{5 / 2}, 2 s_{1 / 2} \\
1 d_{5 / 2}, 2 s_{1 / 2}, 1 d_{3 / 2}\end{array}$ \\
\hline II & ${ }^{12} \mathrm{C}+{ }^{17} \mathrm{O} \rightarrow{ }^{29} \mathrm{Si}$ & 32.21 & $\begin{array}{l}{ }^{12} \mathrm{C} \\
{ }^{17} \mathrm{O} \\
{ }^{29} \mathrm{Si}\end{array}$ & $\begin{array}{l}14.07 \\
12.53 \\
10.49\end{array}$ & $\begin{array}{l}-5.635 \\
-2.500 \\
-1.320\end{array}$ & $\begin{array}{r}1.069 \\
0.272 \\
-0.118\end{array}$ & $\begin{array}{l}45.76 \\
45.76 \\
45.76\end{array}$ & $\begin{array}{l}1 p_{1 / 2}, 1 d_{5 / 2}, 2 s_{1 / 2} \\
1 d_{5 / 2}, 2 s_{1 / 2} \\
1 d_{5 / 2}, 2 s_{1 / 2}, 1 d_{3 / 2}\end{array}$ \\
\hline III & ${ }^{12} \mathrm{C}+{ }^{17} \mathrm{O} \rightarrow{ }^{29} \mathrm{Si}$ & 20.10 & $\begin{array}{l}{ }^{12} \mathrm{C} \\
{ }^{17} \mathrm{O} \\
{ }^{29} \mathrm{Si}\end{array}$ & $\begin{array}{l}8.78 \\
7.82 \\
6.54\end{array}$ & $\begin{array}{l}-2.753 \\
-2.044 \\
-1.320\end{array}$ & $\begin{array}{r}0.587 \\
0.195 \\
-0.118\end{array}$ & $\begin{array}{l}29.65 \\
29.65 \\
31.96\end{array}$ & $\begin{array}{l}1 p_{1 / 2}, 1 d_{5 / 2}, 2 s_{1 / 2} \\
1 d_{5 / 2}, 2 s_{1 / 2}, 1 d_{3 / 2} \\
1 d_{5 / 2}, 2 s_{1 / 2}, 1 d_{3 / 2}, 1 f_{7 / 2}\end{array}$ \\
\hline
\end{tabular}

position of the $1 f_{7 / 2}$ level of ${ }^{29} \mathrm{Si}$ and the $1 p_{1 / 2}$ level of ${ }^{16} \mathrm{O}\left({ }^{17} \mathrm{O}\right)$, which are pushed away from the $s d$ shell with increasing oscillator stiffness. Only in Fig. 4 does the $1 f_{7 / 2}$ level of ${ }^{29} \mathrm{Si}$ have the correct experimental value of $-4.85 \mathrm{MeV}$. In all the level diagrams the $1 p_{1 / 2}$ level of ${ }^{16,17} \mathrm{O}$ is not sufficiently bound. This could only be corrected with a larger stiffness of the two-center oscillator. In summary, the level diagrams represent an optimum fit for all the levels near the Fermi level which are important for our further discussions.

It is important to note that in the shown diagrams the real and avoided crossings of levels occur nearly at the same internuclear distances, independent of the large variations in the parameter

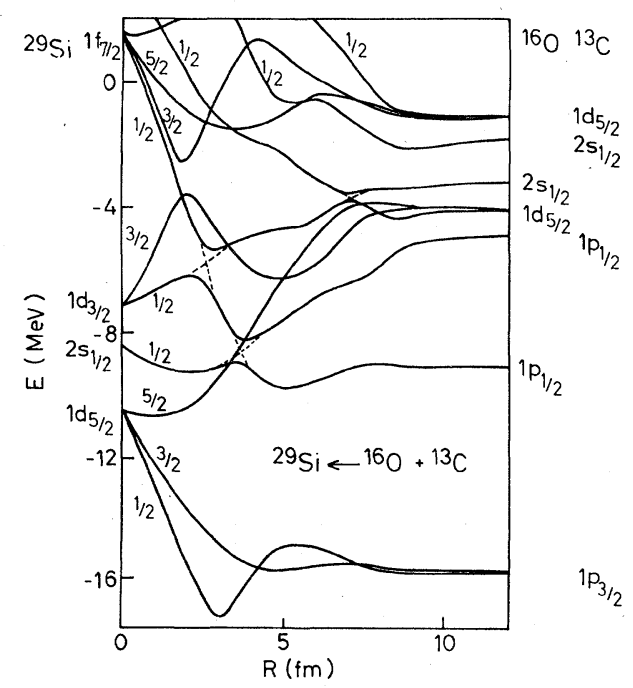

FIG. 2. The neutron levels for ${ }^{16} \mathrm{O}+{ }^{13} \mathrm{C} \rightarrow{ }^{29} \mathrm{Si}$ as functions of the internuclear distance. Avoided level crossings are indicated by dashed lines. For large internuclear distances one recognizes the single-particle spectra of ${ }^{16} \mathrm{O}$ and ${ }^{13} \mathrm{C}$. The parameters are given by set I of Table II. sets of the ATCSM. The avoided crossings, called pseudocrossings, are caused by the mutual repulsion of energy levels having the same quantum number $\Omega$ of the $z$ component of angular momentum. As shown by von Neumann and Wigner ${ }^{8}$ crossings of levels of the same symmetry class are highly improbable.

\section{REACTIONS VIA LEVEL CROSSINGS}

Reaction theories for molecular single-particle motion were first developed in atomic physics. ${ }^{9,10}$ Also for nuclear reactions several authors have studied the excitation of molecular single-particle states by the relative motion of the nuclei. ${ }^{11-17}$ The level diagrams, discussed in the previous section, are prerequisites for molecular reaction theories. Since coupled channel calculations with

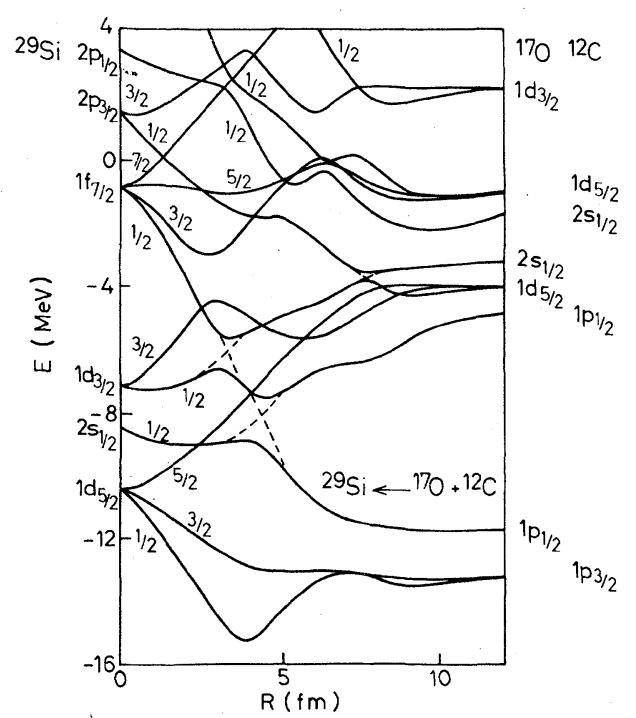

FIG. 3. The neutron levels for ${ }^{17} \mathrm{O}+{ }^{12} \mathrm{C} \rightarrow{ }^{29} \mathrm{Si}$, calculated with the parameters of set II of Table II. 


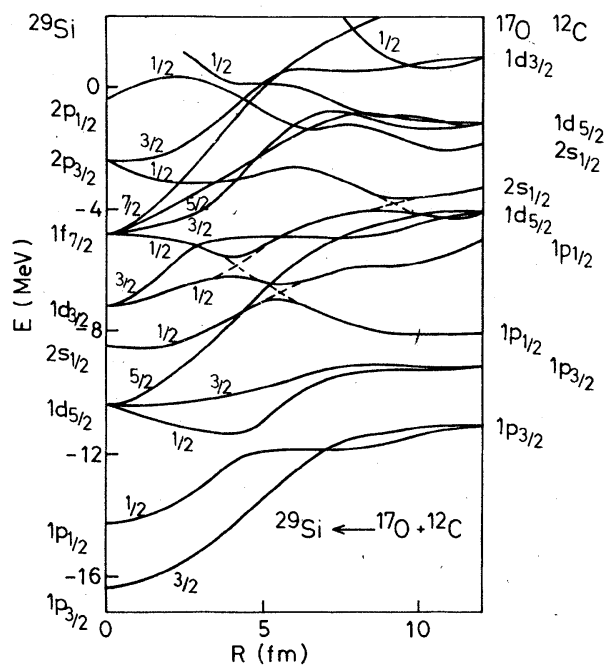

FIG. 4. The neutron levels for ${ }^{17} \mathrm{O}+{ }^{12} \mathrm{O} \rightarrow{ }^{29} \mathrm{Si}$, calculated with the parameters of set III of Table II.

the complete molecular Hamiltonian are tedious, we first select the important reaction channels which are enhancea by the promotion process at points of real and avoided level crossings.

Two velocity-dependent excitation mechanisms are possible between molecular single-particle states $^{9}$ :

(a) The rotational coupling interacts between levels with $\Omega$ and $\Omega \pm 1$, where $\Omega$ is the quantum number of the component of angular momentum in the direction of the internuclear axis,

$$
\left\langle\Omega\left|j_{ \pm}\right| \Omega \mp 1\right\rangle \text {. }
$$

Here $j_{t}$ is the operator of angular momentum of the single particle.

(b) The radial coupling causes transitions near avoided crossings between levels with equal values of $\Omega$ :

$$
\left\langle\Omega\left|\frac{\partial}{\partial R}\right| \Omega\right\rangle
$$

Let us discuss the possible consequences of level crossings for the ${ }^{13} \mathrm{C}+{ }^{16} \mathrm{O}$ scattering, where the loosely bound neutron first occupies the $1 p_{1 / 2}$ level. From Fig. 2 we note that this level has an avoided crossing with the $1 p_{1 / 2}$ level of ${ }^{16} \mathrm{O}$ near 3-4 fm. Therefore, a neutron in the $1 p_{1 / 2}$ level of ${ }^{16} \mathrm{O}$ may be promoted to the $1 p_{1 / 2}$ level of ${ }^{13} \mathrm{C}$, thus causing a neutron transfer from ${ }^{16} \mathrm{O}$ to ${ }^{13} \mathrm{C}$, i.e., ${ }^{13} \mathrm{C}+{ }^{16} \mathrm{O} \rightarrow{ }^{14} \mathrm{C}+{ }^{15} \mathrm{O}$. A further avoided crossing of the $1 p_{1 / 2}$ level of ${ }^{13} \mathrm{C}$ with the $1 d_{5 / 2}$. level with $\Omega=\frac{1}{2}$ of ${ }^{16} \mathrm{O}$ occurs near $2-4 \mathrm{fm}$. The latter level has a second avoided crossing near
6-8 $\mathrm{fm}$ with the $2 s_{1 / 2}$ level of ${ }^{17} \mathrm{O}$ (see Figs. 3 and 4). Therefore, both crossings give rise to the neutron transfer ${ }^{13} \mathrm{C}+{ }^{16} \mathrm{O} \rightarrow{ }^{12} \mathrm{C}+{ }^{17} \mathrm{O}$, where the ${ }^{17} \mathrm{O}$ nucleus is left in the ground or first excited state. Both cross sections have been experimentally observed. ${ }^{18}$ The rotational coupling plays a role between the $1 p_{1 / 2}$ level of ${ }^{13} \mathrm{C}$ with $\Omega=\frac{1}{2}$ and the $1 d_{5 / 2}$ level of ${ }^{17} \mathrm{O}$ with $\Omega=\frac{3}{2}$ near $5-8 \mathrm{fm}$ and enhances the neutron transfer ${ }^{13} \mathrm{C}+{ }^{16} \mathrm{O}-{ }^{12} \mathrm{C}+{ }^{17} \mathrm{O}$ (g.s.).

We expect that the cross sections, obtained in the framework of coupled channel calculations, will reveal specific signatures of the avoided and real level crossings as functions of the incident energy. Mainly, level crossings which exist in the range of 4-7 fm of the internuclear distance have a real chance to be observable. The level crossings, which are located more inside, may be hidden by the strong absorptive processes occurring at smaller internuclear distances.

\section{CONCLUDING REMARKS}

The enhanced excitation near points of level crossings is a specific molecular effect. It should be observable in the excitation function for inelastic scattering and transfer of the nucleus. Such an enhancement cannot easily be described in a basis of states centered around a single nucleus. The states of TCSM already include the $R$ dependent deformation of the nuclear shape, whereas the one-center states have first to be superposed in order to describe the change of the shell model potential due to the second nucleus. Therefore, we believe that a convincing experimental proof for molecular single-particle orbitals may be found in the search for structures in the energy dependence of certain cross sections, where level crossings are expected to contribute.

In this work each fragmentation, e.g., ${ }^{13} \mathrm{C}+{ }^{16} \mathrm{O}$ and ${ }^{12} \mathrm{C}+{ }^{17} \mathrm{O}$ is described with an individual TCSM having different parameters. It would be worthwhile to develop a hybrid TCSM with a single parameter set, which describes both fragmentations in neutron transfer reactions. In the case of the reaction ${ }^{13} \mathrm{C}+{ }^{16} \mathrm{O} \rightarrow{ }^{12} \mathrm{C}+{ }^{17} \mathrm{O}$ the hybrid TCSM would yield the ${ }^{13} \mathrm{C}$ and ${ }^{17} \mathrm{O}$ states for large internuclear distances. For $r \rightarrow 0$ it would approach the spectrum of ${ }^{29} \mathrm{Si}$. The reaction theory with such a TCSM can be formulated in analogy with methods used in molecular chemistry and outlined in Ref. 19.

Coupled channel calculations for cross sections, applying the level diagrams we have discussed and the methods outlined in Refs. 16 and 17, are in progress. 


\section{ACKNOWLEDGMENTS}

We gratefully acknowledge discussions with Professor N. Gruen and G. Terlecki. The topic of the nuclear promotion process was brought to our attention by Professor Dr. Ch. Toepffer. This work was supported by the Deutsche Forschungsgemeinschaft, Gesellschaft für Schwerionenforschung (GSI) and Bundesministerium fuer Forschung und Technologie.
${ }^{1}$ P. Holzer, U. Mosel, and W. Greiner, Nucl. Phys. A138, 241 (1969); D. Scharnweber, U. Mosel, and W. Greiner, ibid. A164, 257 (1971).

${ }^{2} \mathrm{~K}$. Pruess and W. Greiner, Phys. Lett. 33B, 197 (1970); U. Mosel, T. D. Thomas, and P. Riesenfeldt, ibid. 33B, 565 (1970).

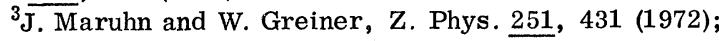
M. G. Mustafa, U. Mosel, and H. W. Schmitt, Phys. Rev. Lett. 28, 1536 (1972); Phys. Rev. C 7]; 1519 (1973).

${ }^{4}$ W. von Oertzen and H. F. Bohlen, Phys. Rep. 19C, 1 (1975).

${ }^{5}$ L. Landau, Phys. Z. Sow. 2 46 (1932); C. Zener, Proc. R. Soc. A137, 696 (1932).

${ }^{6}$ U. Fano and W. Lichten, Phys. Rev. Lett. 14, 627 (1965); W. Lichten, Phys. Rev. 164, 131 (1967); M. Barat and W. Lichten, Phys. Rev. A 6, 211 (1972).

${ }^{7}$ D. Glas and U. Mosel, Phys. Lett. 49B, 301 (1974); Nucl. Phys. A264, 268 (1976).

${ }^{8} \mathrm{~J}$. von Neumann and E. Wigner, Phys. Z. 30, 467 (1929).

${ }^{9}$ B. H. Bransden, Atomic Collision Theory (Benjamin, New York, 1970) and references therein.

${ }^{10}$ J. S. Briggs, Rep. Prog. Phys. 39, 217 (1976).
${ }^{11}$ J. Y. Park, W. Scheid, and W. Greiner, Phys. Rev.

C $\underline{6}, 1565$ (1972).

${ }^{12}$ W. von Oertzen and W. Nörenberg, Nucl. Phys. A207, 113 (1973).

${ }^{13}$ F. Becker, S. Joffily, C. Beccaria, and G. Baron, Nucl. Phys. A221, 475 (1974).

${ }^{14}$ M. G. Mustafa, Phys. Rev. C 14, 2168 (1976).

${ }^{15}$ G. Delic, K. Pruess, L. A. Charlton, and N. K. Glendenning, Phys. Lett. 69B, 20 (1977); K. Pruess, Nucl. Phys. A278, 124 (1977); K. Pruess and P. Lichtner, ibid. A291, 475 (1977).

${ }^{16} \mathrm{G}$. Terlecki, W. Scheid, H. J. Fink, and W. Greiner, Phys. Rev. C 18, 265 (1978).

${ }^{17}$ J. Y. Park, W. Scheid, and W. Greiner, Phys. Rev. C 20, 188 (1979).

${ }^{18} \mathrm{~W}$. Bohne et al., report, Hahn-Meitner-Institut, Berlin, 1979.

${ }^{19}$ W. R. Thorson and J. B. Delos, Phys. Rev. A 18,117 (1978); 18, 135 (1978).

${ }^{20} \mathrm{~F}$. Ajz enberg-Selove, Nucl. Phys. A268, 1 (1976); A281, 1 (1977); B. H. Wildenthal and J. B. McGrory, Phys. Rev. C 7, 714 (1973); P. M. Endt and C. van der Leun, Nucl. Phys. A310, 1 (1978). 